III-V nanowire arrays: growth and light interaction

This content has been downloaded from IOPscience. Please scroll down to see the full text. 2014 Nanotechnology 25014015

(http://iopscience.iop.org/0957-4484/25/1/014015)

View the table of contents for this issue, or go to the journal homepage for more

Download details:

This content was downloaded by: annafm

IP Address: 128.178.106.14

This content was downloaded on 17/12/2013 at 05:36

Please note that terms and conditions apply. 


\title{
III-V nanowire arrays: growth and light interaction
}

\section{Heiss $^{1}$, E Russo-Averchi ${ }^{1}$, A Dalmau-Mallorquí ${ }^{1}$, G Tütüncüoğlu ${ }^{1}$, F Matteini, D Rüffer, S Conesa-Boj, O Demichel, E Alarcon-Lladó and A Fontcuberta i Morral}

\author{
Laboratory of Semiconductor Materials, Ecole Polytechnique Fédérale de Lausanne, 1015 Lausanne, \\ Switzerland \\ E-mail: anna.fontcuberta-morral@epfl.ch
}

Received 3 July 2013, in final form 17 August 2013

Published 11 December 2013

\begin{abstract}
Semiconductor nanowire arrays are reproducible and rational platforms for the realization of high performing designs of light emitting diodes and photovoltaic devices. In this paper we present an overview of the growth challenges of III-V nanowire arrays obtained by molecular beam epitaxy and the design of III-V nanowire arrays on silicon for solar cells. While InAs tends to grow in a relatively straightforward manner on patterned (111)Si substrates, GaAs nanowires remain more challenging; success depends on the cleaning steps, annealing procedure, pattern design and mask thickness. Nanowire arrays might also be used for next generation solar cells. We discuss the photonic effects derived from the vertical configuration of nanowires standing on a substrate and how these are beneficial for photovoltaics. Finally, due to the special interaction of light with standing nanowires we also show that the Raman scattering properties of standing nanowires are modified. This result is important for fundamental studies on the structural and functional properties of nanowires.
\end{abstract}

(Some figures may appear in colour only in the online journal)

\section{Introduction}

Semiconductor nanowires have been the object of intensive study in the last few years. Their longitudinal geometry and small footprint provide numerous advantages for optoelectronic and energy harvesting and storage devices [1-5]. Additionally, they can be the base for more complex nanostructures such as axial/radial heterostructures, nanotrees, nanocrosses, nanomembranes and nanocubes/diamonds [6-11]. Recently, it has been shown that the relatively small cross-section of the nanowires provides a path for the integration of mismatched materials within a nanowire [12, 13] and also to grow nanowires of a certain material on mismatched substrates [14]. Novel materials combinations have been achieved such as the growth of Si segments in a III-V nanowire [15-17] and III-V nanowires on silicon for high performance electronics $[18,19]$.

The integration of III-V nanowires on silicon is particularly attractive in the area of photonics and photovoltaics [20]. For example, a GaAs nanowire-based solar cell could be grown on a silicon pn junction forming a dual-junction solar

1 These authors contributed equally to this work. cell [21]. Here, nanowires would not only provide the active material for the top cell; they could intrinsically provide an antireflective layer. Several works have shown that the arrangement of nanowires in a nanowire-based solar cell is extremely important for both the collection of light in the wires and for an isotropic reduction of the reflectivity [22, 23]. Recently, studies on single and multiple nanowire-based solar cells have shown photonic effects which lead to a several-fold broadband increased absorption in each nanowire. In particular, the Lund group has obtained a conversion efficiency of $13.8 \%$ under $1.5 \mathrm{AM}$ illumination conditions by optimizing the nanowire array configuration [23]. Along the same lines, we have recently shown that light absorption in a single nanowire solar cell can be enhanced more than one order of magnitude due to a self-concentrating effect [24]. To the best of our knowledge, the effect on the nanowire dimensions and density on a substrate have not been investigated in detail yet.

In this work, we present some recent results on the gold-free growth of III-V nanowire arrays and on the interaction of light with the nanowires of the arrays. We highlight some of the existing challenges for the understanding and for the reproducible growth of nanowires in the array form. Next, we turn to the application of nanowire 
arrays. We start presenting finite-difference-time-domain simulations on the light absorption of III-V nanowire arrays as a function of the inter-wire distance and show some results on Raman spectroscopy of nanowires as a function of the nanowire spacing. It is particularly interesting that the self-concentration effect in nanowire results in a variation of the Raman selection rules and opens up possibilities in the area of light scattering with nanowire-based nanostructures.

\section{Growth of III-V arrays on silicon}

Organized growth of nanostructures on a substrate is the first step towards the rational and parallel fabrication of devices. In addition, by growing nanowires on precise locations and with controlled positioning should be the ideal setting for studying their growth mechanisms. The most common way of organizing growth of nanowires on a substrate is to pattern a substrate either with gold nano-droplets or with nanoscale holes on a silicon dioxide mask. Nanoscale openings in a silicon dioxide mask provide a preferential site for nanowire for catalyst-free growth methods. However, given the same growth conditions, growth dynamics of nanowires depends in a high degree on their dimensions and center-to-center distance [25-29]. The nanoscale features for nanowire growth can be defined on a substrate by electron beam, nanosphere, phase-shift or nano-imprint lithography [30-34].

III-V nanowires grow mostly along the (111)B direction [35, 36]. This represents a challenge for growing on (111) silicon, as the polarity at the interface needs to be type B for the nanowires to grow perpendicularly to the substrate [37-42]. It is interesting to compare the catalyst-free growth of InAs and GaAs on silicon: while GaAs nanowires grow assisted by a Ga droplet at their tips, InAs nanowires seem to grow without the assistance of an In droplet by selective area epitaxy [43-45]. At the same time, InAs nanowire arrays are relatively straightforward to grow, while GaAs encounter many more challenges [46-49].

Nanowire arrays were grown by molecular beam epitaxy (MBE) in a DCA P600 system without the use of a predefined metal particle. In order to localize the growth on the substrate, thermally oxidized silicon wafers were patterned with nanoscale holes. The holes were defined by electron beam lithography and wet-chemical etching based on 7:1 buffered hydrofluoric acid solution. In order to ensure an oxide-free surface in the holes, the wafers were shortly dipped in the same HF solution prior to growth. They were subsequently kept in isopropanol until the loading in the MBE to preserve the cleanliness of the substrate. The patterns consisted in a square arrangement of holes of nominal sizes ranging between 90 and $150 \mathrm{~nm}$. The inter-hole distance or pitch was varied between 200 and $2000 \mathrm{~nm}$ on the same substrate. The oxide thickness was $20 \mathrm{~nm}$ for the InAs growth and was varied between 5 and $30 \mathrm{~nm}$ for the GaAs.

\section{1. $\ln A s$}

InAs nanowires on patterned silicon substrates were grown at a nominal In growth rate of $0.2 \AA \mathrm{s}^{-1}, \mathrm{As}_{4}$ partial pressure of $6.0 \times 10^{-6}$ Torr (BEP: beam equivalent pressure) and substrate temperature of $500^{\circ} \mathrm{C}$ for $1 \mathrm{~h}$. Scanning electron micrographs (SEM) of InAs nanowire arrays grown on a $\mathrm{Si}(111)$ substrate are shown in figures $1(\mathrm{a})-(\mathrm{g})$. In the context of InAs nanowire growth, it is well known that a tradeoff exists between the growth selectivity and the final length of the nanostructures [46]: this has recently also been observed in the case of InAs V-shaped membranes [50]. The selectivity of growth in the openings of the substrates improves with the temperature until parasitic growth on $\mathrm{SiO}_{2}$ completely disappears. Indeed, the sticking probability of indium ad-atoms on oxide decreases by increasing the temperature leading to the nucleation of nanowires only in the predefined holes. However, the higher is the temperature the higher is desorption of the deposited material and thus the shorter the structures grown. This means that the ideal growth conditions represent a compromise between optimal selectivity and maximum length, and these are the ones we adopted in this study. Within these growth conditions we could achieve high selectivity and aspect ratio, which were needed for the optical measurements. The nanowire growth yield, here defined as number of openings nucleating nanowires divided by the total number of openings in the pattern, was $92 \%$.

The sample reported in figure 1 shows a significant size variation of the nanowires as a function of the inter-hole distance (pitch). In particular the diameter and length saturates around a value of 155 and $1480 \mathrm{~nm}$ for pitch $800 \mathrm{~nm}$. A similar behavior has been observed by other groups in the case of ordered growth of InAs nanowires [44, 46, 51] and nanomembranes [50]. The dependence of the nanowire length as a function of the pitch can be explained by the existence of two separate growth regimes: (i) a competitive growth regime with shorter nanowires for narrow inter-holes distances and (ii) a diffusion-limited or independent growth regime for wider pitches. The indium surface diffusion length on $\mathrm{SiO}_{2}\left(\lambda_{\mathrm{SiO}_{2}}\right)$ determines the switch between the two regimes. Indeed, $\lambda_{\mathrm{SiO}_{2}}$ limits the surface collection area i.e. the sample area from which each wire can collect the In species diffusing on the oxide. For a small nanowire spacing (pitch $<2 \lambda_{\mathrm{SiO}_{2}}$ ), the indium ad-atoms are shared between the wires. By increasing the spacing, the surface collection area available exclusively to each nanowire increases, resulting in a linear increase of the growth rate. In the opposite limit of a large spacing (pitch $>2 \lambda \mathrm{SiO}_{2}$ ) the wires can be treated as independent isolated islands. In this regime the surface collection area cannot increase anymore and the growth rate is no longer dependent on the pitch. The calculated volume of the nanowires depends on the pitch and it can be seen that it saturates when the growth reaches the diffusion-limited regime (see figure 1(e)). For completeness, in figure 1(f) we show the equivalent layer thickness, defined as the thickness of the equivalent layer obtained with the same InAs volume. This will be useful for the analysis of the Raman scattering data in the arrays, as discussed later in this work.

\subsection{GaAs}

We turn now to the study of Ga-assisted growth of GaAs nanowires on $\mathrm{SiO}_{2} / \mathrm{Si}$ patterned substrates. In this 


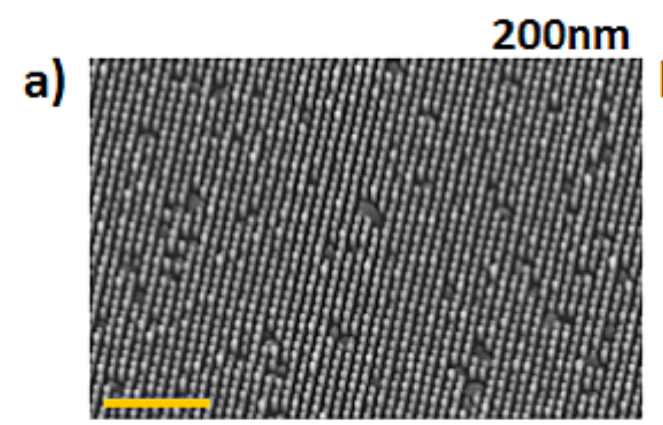

$800 \mathrm{~nm}$

c)

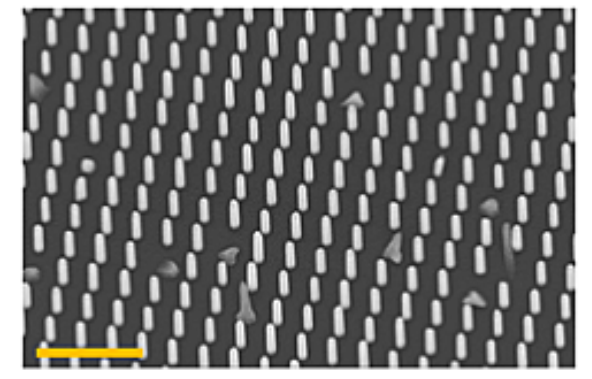

b)

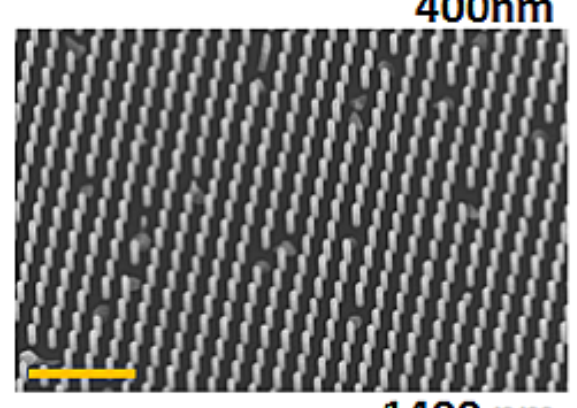

$1400 \mathrm{~nm}$

d)
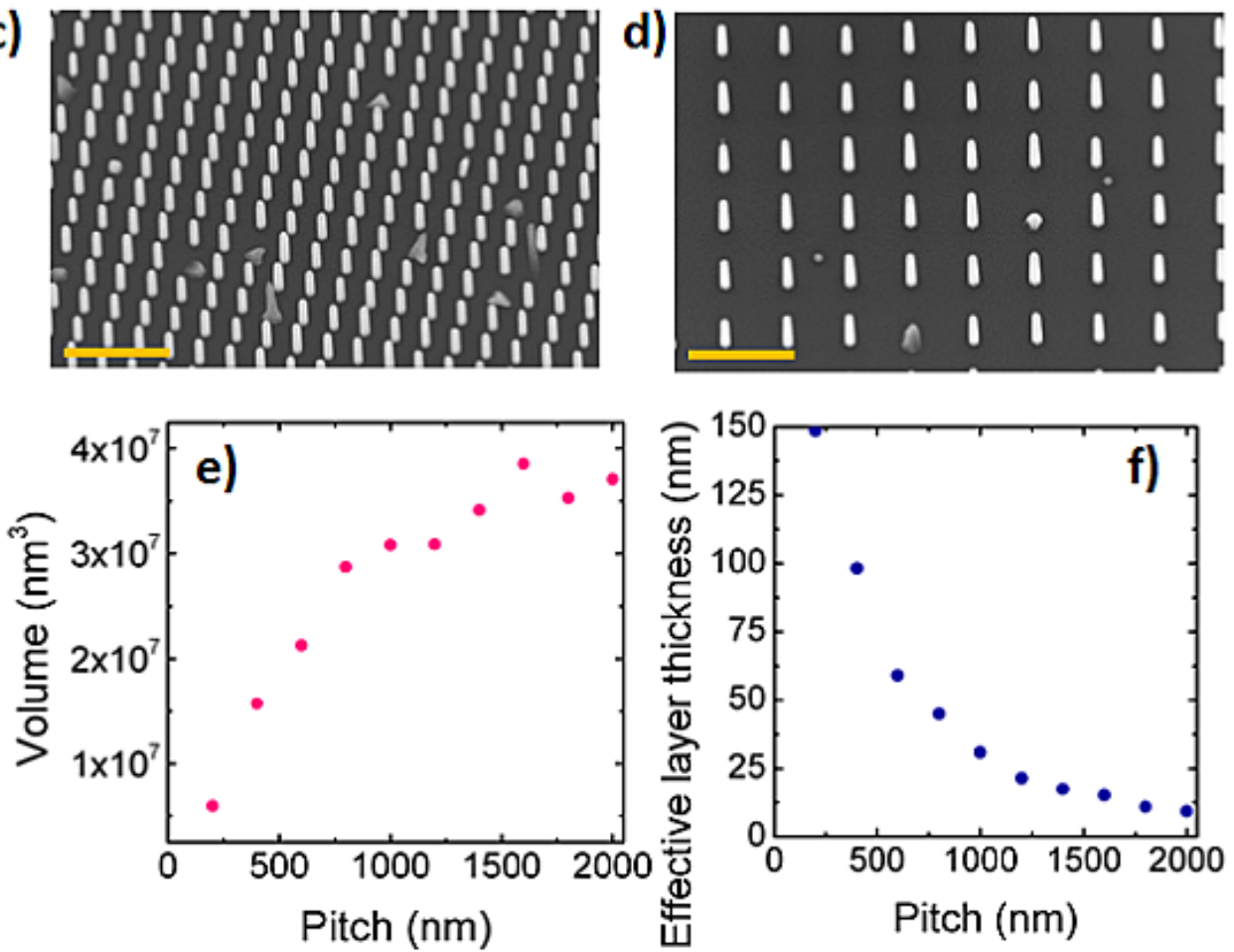

Figure 1. (a)-(d) Scanning electron micrographs of InAs nanowires obtained on a patterned Si substrate for different pitch (scale is $2 \mu \mathrm{m}$ ), (e) nanowire volume $\left(V_{\mathrm{NW}}\right)$ and (f) effective layer thickness as a function of the pitch.

case, a nanoscale $\mathrm{Ga}$ droplet gathers preferentially $\mathrm{As}_{4}$ molecules to drive the GaAs growth. The Ga droplet is continuously replenished by the diffusion of Ga-adatoms from the facets and surface for ensuring the continuous nanowire growth [52]. Recently, it has been shown that the substrate preparation and the pre-growth conditioning are key to achieve high-yield growth of GaAs nanowire arrays by the Ga-assisted method [49]. We have investigated two of these main factors, namely the degassing of the sample prior to growth and the thickness of the growth mask layer. Figure 2 shows a sequence of $20^{\circ}$ tilted SEM images of the nanowire arrays obtained in this study. The GaAs nanowires grown on patterned $\mathrm{Si}(111)$ substrates, with a nominal Ga growth rate of $1 \AA \mathrm{s}^{-1}, \mathrm{As}_{4}$ partial pressure of $2 \times 10^{-6}$ Torr, substrate temperature of $615^{\circ} \mathrm{C}$, and with $7 \mathrm{rpm}$ substrate rotation. In all cases the samples have been degassed at $600^{\circ} \mathrm{C}$ for $2 \mathrm{~h}$ and they have been all grown with the same growth recipe. Figure 2(a) shows the results of the first growth: the yield of vertical wires is low and parasitic bulk growth occurs. The sample reported in figure 2(b) has been grown after a further heating at $770{ }^{\circ} \mathrm{C}$ for $30 \mathrm{~min}$ in order to remove any possible contaminants of the surface: the density of vertical wires is significantly improved, thus demonstrating that the sample preparation and the cleaning of the surface are decisive steps in obtaining high quality nanowire arrays.

Subsequently, we evaluated the influence of thickness of the oxide layer on nanowire growth yield. The thickness of the oxide layer was determined with spectroscopic ellipsometry prior to growth. Growths of GaAs nanowires on arrays with $\mathrm{SiO}_{2}$ layers of 13,18 and $21 \mathrm{~nm}$ thickness are reported in figures 2(c)-(i). As also found by Plissard et al [49], by decreasing the oxide thickness the yield improves to dramatically drop again at thinner thicknesses. We found an optimal oxide thickness of $18 \mathrm{~nm}$, which is not affected by the pitch of the array. The geometry of the pattern is found to affect in itself the yield of vertical wires, an observation which needs further investigation. The maximum yield (65\%) is reached for pitch $=400 \mathrm{~nm}$ and opening size $=100 \mathrm{~nm}$ (figure 2(c)). 

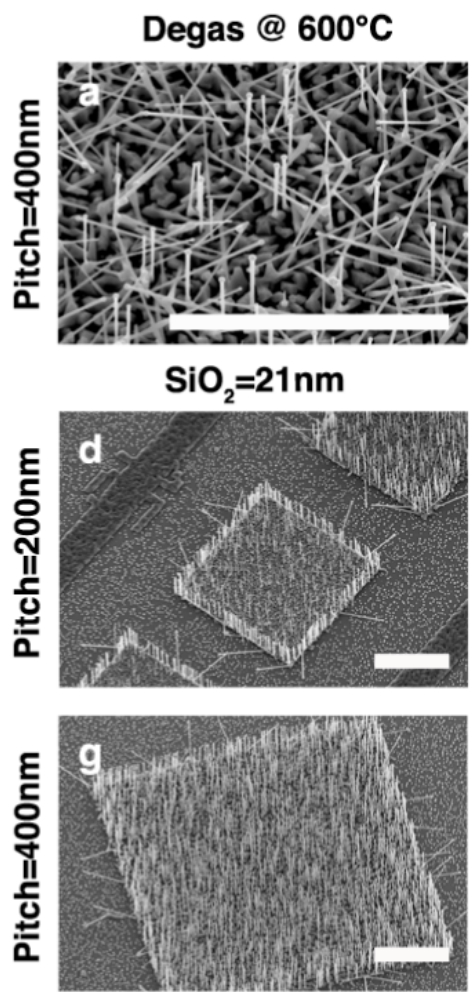

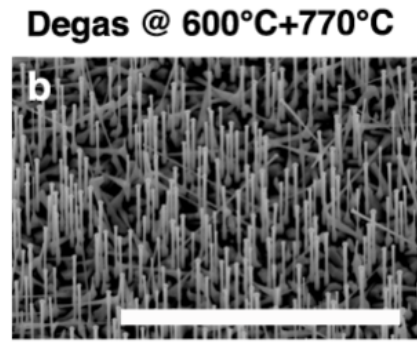

$\mathrm{SiO}_{2}=18 \mathrm{~nm}$

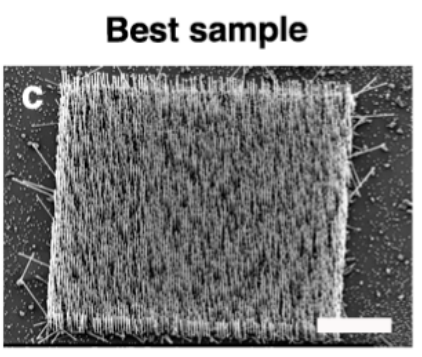

$\mathrm{SiO}_{2}=13 \mathrm{~nm}$
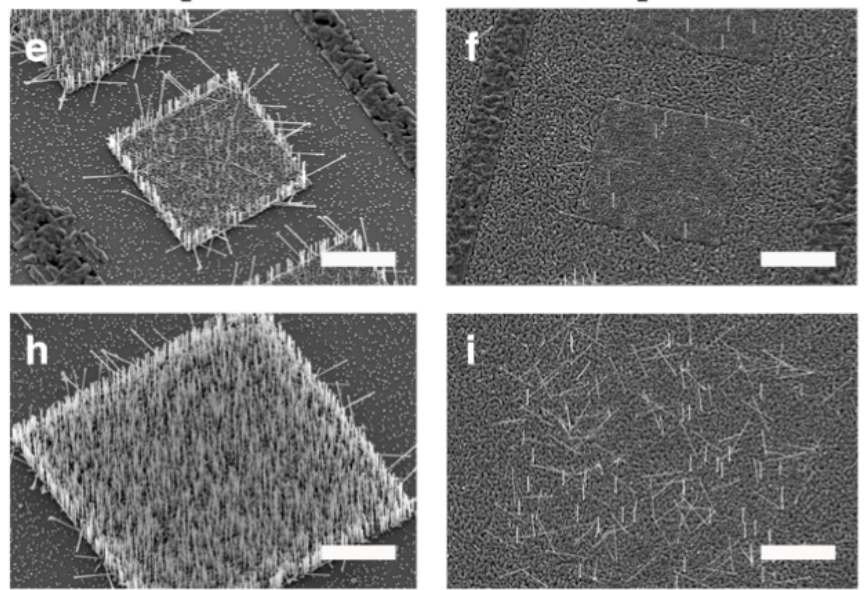

Figure 2. Scanning electron micrographs of GaAs nanowires obtained on patterned (111)Si substrates as a function of the oxide thickness, pitch and substrate preparation conditions (see text).

Further optimization can be achieved by studying several other parameters like the substrate temperature, the $\mathrm{V} / \mathrm{III}$ ratio and the catalyst pre-deposition. However we have encountered several issues in the long-term reproducibility of the samples. In particular, we have recently observed that small changes in the surface roughness of the substrates results in extremely different nanowire density and orientation on a silicon substrate [53]. We believe this kind of variation from batch to batch might be one of the sources for lack of reproducibility for the Ga-assisted growth of GaAs nanowire arrays.

\section{Light interaction with III-V nanowire arrays}

We turn now our attention towards understanding of how a planar light wave incident perpendicularly to the substrate interacts with standing nanowires. As mentioned above, we have recently shown a self-concentration effect occurring in single nanowires standing on a substrate [24]. Here below we present calculations on light absorption in standing nanowires, and we show how this changes in an array as a function of the inter-wire distance. Finally, we show how this concentration effect affects the Raman spectra of nanowires.

\subsection{Light absorption in standing nanowires}

Light absorption in standing GaAs nanowires of different diameters surrounded by vacuum was calculated by solving the wave equation for an incident radiation propagating along the nanowire axis. For this, we use finite-difference-timedomain simulations [54], similar to what the Witzigmann group has been realized recently [55]. We start by presenting the propagation of a planar wave impinging vertically on a $2 \mu \mathrm{m}$ long GaAs nanowire. We have calculated the spectral and diameter dependence of the absorption rate. The enhancement in absorption for a single standing nanowire is defined as the ratio between the absorbed power and the power of the incident electromagnetic radiation on the nanowire surface ( $S=\pi r^{2}$, where $r$ is the nanowire radius):

$$
\text { Absorption enhancement }=\frac{P_{\text {absorbed }}}{P_{\text {inc }} S}
$$

where $P_{\text {absorbed }}$ is the power absorbed by the nanowire and $P_{\text {inc }}$ is the incident power surface density. Clearly, this efficiency will be larger than $100 \%$ in the cases where the absorption cross-section is larger than the apparent one defined by the physical limits of the nanowire. The spectral dependence of the absorption enhancement as a function of the nanowire diameter is plotted in figure 3(a). The color scale denotes the value of the enhancement. The graph is composed of two main branches of enhanced absorption. The first one, defined by an enhancement of the spectral absorption close to 70 fold, corresponds to nanowires between 50 and $150 \mathrm{~nm}$. It is important to note that the absorption enhancement is always higher than 10 in the 300-900 $\mathrm{nm}$ range, where GaAs absorbs at room temperature. The second branch corresponds to an enhancement of 10 fold in the absorption for diameters above $150 \mathrm{~nm}$. These both branches correspond to resonances for which the light is better trapped in the nanowire structure. 

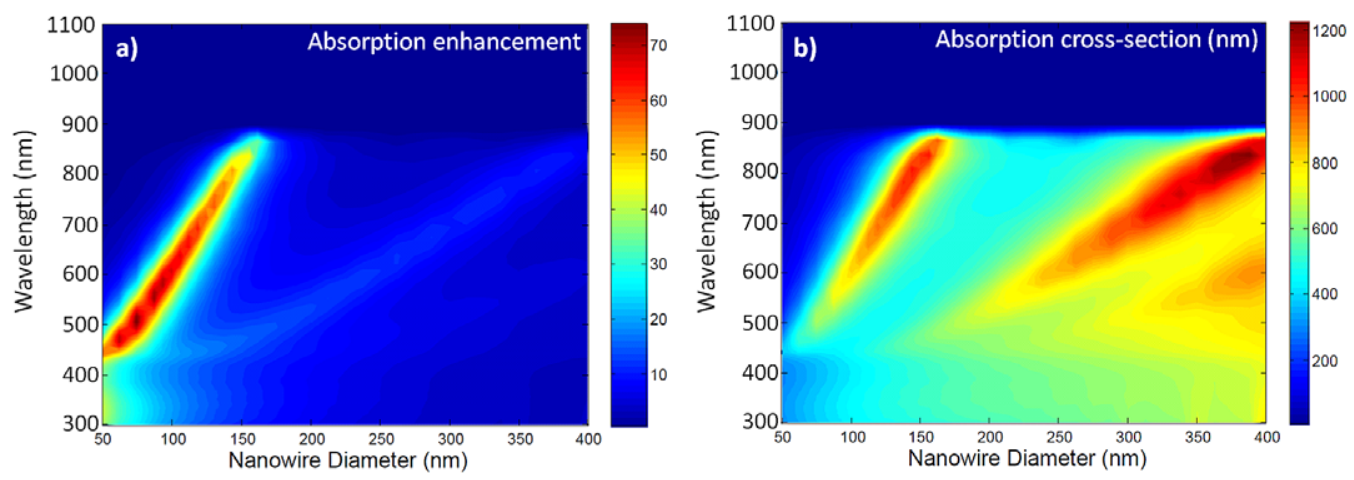

Figure 3. (a) Absorption enhancement and (b) diameter of the absorption cross-section of single vertical GaAs nanowires standing on a Si substrate as a function of the diameter.

Along these branches, the resonant wavelength increases with the nanowire diameter. It is interesting to note that there is at least a four-fold increase of the absorption in the whole spectrum even for larger nanowires (diameter above $100 \mathrm{~nm}$ ). This represents a significant broadband absorption increase, difficult to observe in lying nanowires [56-59].

A way of capturing the origin of the dramatic increase in light absorption, we proceed by calculating the absorption cross-section, defined by the disk of radius $r^{*}$ corresponding to the equivalent area under which the absorbed power density $P_{\mathrm{abs}} / \pi r^{* 2}$ is equal to the incident surface power density, $P_{\text {inc }}$ :

$$
r^{*}=\sqrt{\frac{P_{\mathrm{abs}}}{\pi P_{\mathrm{inc}}}} .
$$

In figure 3(b) we plot the spectral dependence of the capture cross-section diameter $\left(2 r^{*}\right)$ as a function of the nanowire diameter. In a thin film the absorption cross-section has identical dimensions to its size, while in the case of nanoscale objects the cross-section can be quite larger [60,61]. We find cross-sections as large as $1200 \mathrm{~nm}$ for the longest wavelengths $(830 \mathrm{~nm})$ and largest nanowires. Interestingly, the plot of the cross-sections diameter amplifies the branches found in the absorption efficiency mapping. One of the consequences of the large absorption cross-section in standing nanowires is that in a solar cell they should be disposed at an optimized distance. At the same time, the self-concentrating effect should result in an increase of the device efficiency and provides a path for surpassing the efficiency limits posed by Shockley and Queisser in 1961 as explained in the [24, 62].

We direct now our discussion towards the interaction of light in nanowire arrays as a function of the inter-wire distance or pitch. We have extended our calculations to nanowire forming square arrays on silicon and exhibiting a diameter of $150 \mathrm{~nm}$. For simplicity in the calculations we have assumed a square array. In a real nanowire-based solar cell device, the arrangement should be optimized so that the nanowires are separated by the optimum pitch and avoid any possible diffraction effects $[63,64]$. Figure 4(a) depicts the square of the electric field of light propagating in the nanowire (which is proportional to the light intensity), weighted by the solar spectrum 1.5 AM as a function of the pitch. The smallest pitch is $150 \mathrm{~nm}$, which corresponds to the thin film case.
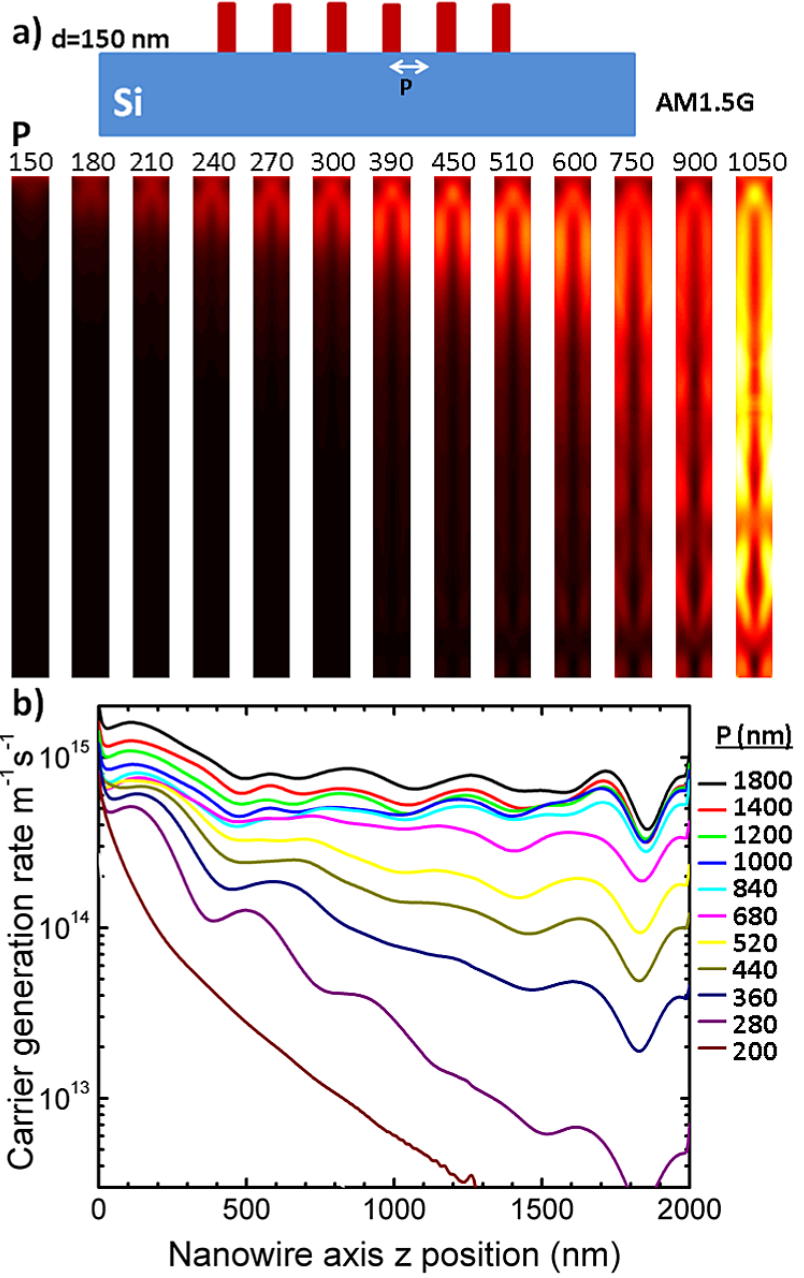

Figure 4. Calculations of light absorption in GaAs nanowires in an array in AM 1.5 conditions. (a) Average of the square of the electric field in the nanowire for different pitch, (b) profile of the carrier generation along the nanowire axis as a function of the pitch.

In this case, the light intensity decays exponentially at the top of the nanowire/thin film. By separating the nanowires the light starts to be present to a deeper length within the nanowire. For a pitch of $750 \mathrm{~nm}$ a significant amount of light is able to propagate till the bottom of the nanowire. 
The intensity of light along the nanowire axis increases by further separating the nanowires. One should note that the distribution of the intensity along the nanowire seems to exhibit some nodes, showing that the absorption enhancement corresponds to a resonance and that the nanowire acts as a sort of cavity. From the distribution of the square of the electric field along the nanowire we calculate the overall carrier generation rate along the nanowire axis. The results are shown in figure 4(b). In agreement with the distribution of the light intensity, the generation of carriers increases and homogenizes along the nanowire axis when the pitch of the nanowire array increases. Interestingly, the carrier generation does not follow the exponential decay from Lambert-Beer law but a relatively homogeneous absorption along the nanowire axis. This distribution of light absorption is at the same time more beneficial for carrier extraction in the radial pn junction design. Along with the self-concentration effect, the generation of carriers all along the nanowire axis could lead to devices with a higher $V_{\mathrm{OC}}$ and a smaller series resistance. Overall this would result in a higher photo-conversion efficiency.

\subsection{Raman scattering in standing InAs nanowire arrays}

Backscattering Raman measurements were conducted using the $488 \mathrm{~nm}$ line of an $\mathrm{Ar} / \mathrm{Kr}^{+}$gas laser focused on the InAs arrays, described in section 2.1, through a 0.75 N.A. microscope objective. The total laser power for the experiments was kept at $300 \mu \mathrm{W}$. The Raman scattered light was projected on the entrance slit of a triple grating spectrometer and collected by means of a charge coupled device. Experiments were conducted on a series of as-grown nanowires obtained during a single MBE growth run on a sample with patterned fields of varying inter-wire spacing. Figure 5(a) shows a typical Raman spectrum of the InAs nanowires obtained under such conditions. One can observe two modes at 211 and $236 \mathrm{~cm}^{-1}$, reported respectively as TO and LO for InAs. Still, the exact position of these modes depends on the existence of zinc-blende, wurtzite and/or twining defects [65-68]. We also observe a mode at $520 \mathrm{~cm}^{-1}$ resulting from the silicon substrate. Figure 5(b) shows the absolute intensities of these modes in dependence of nanowire array spacing. One can see that while the absolute intensities of InAs related modes decrease with nanowire spacing the relative intensity of the silicon mode is increasing.

Figure 5(c) depicts the top InAs surface coverage and equivalent thin film volume normalized to the values extrapolated for $0 \mathrm{~nm}$ pitch (thin film case). As seen in figure 5(b), for small pitch distances, both TO and LO decrease in intensity, which is explained by the loss of InAs scattering cross-section because of the lower nanowire density in the probing area. Interestingly, for pitches larger than the laser spot diameter (around $800 \mathrm{~nm}$ ), the LO intensity reaches a plateau and even increases slightly with pitch distance, thus indicating that an effect beyond the purely geometric material decrease plays a role. Actually, the signal seems to correlate with the volume of a single nanowire from figure 5(c), fact that suggests a light coupling effect that allows the light to probe
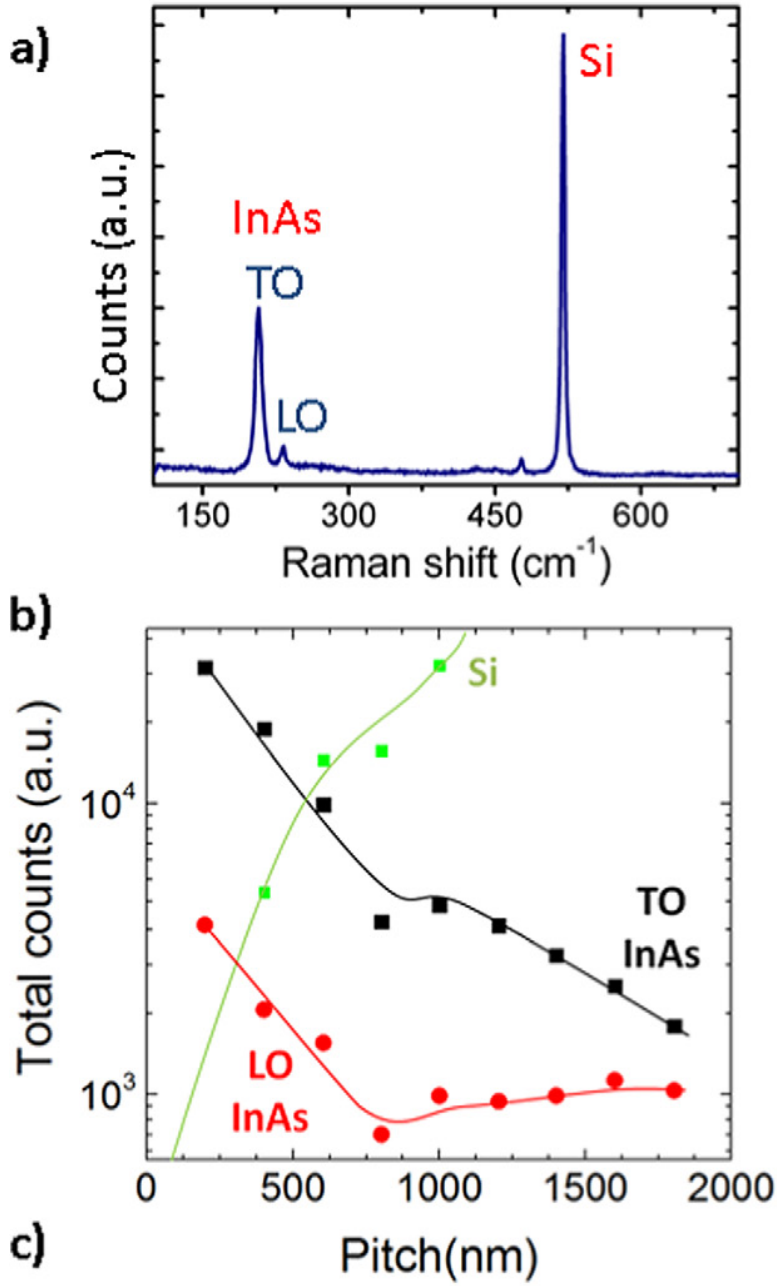

b)

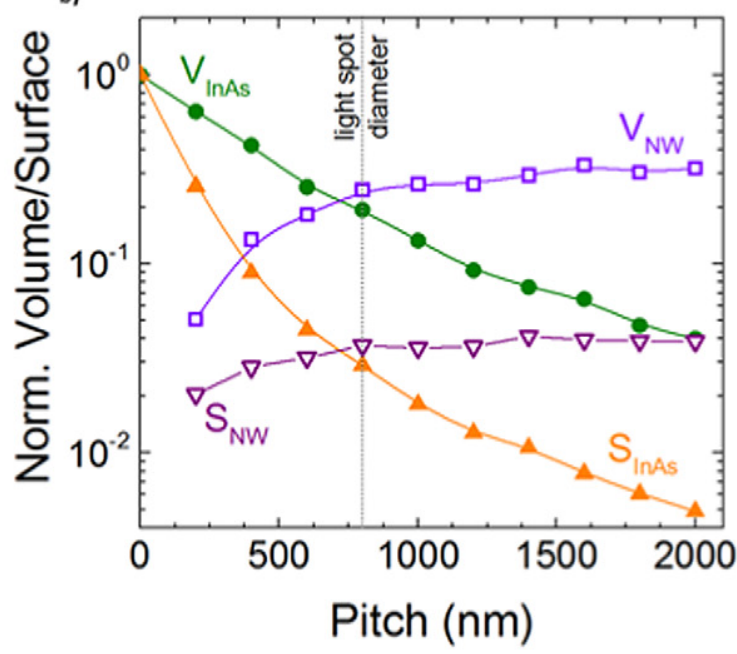

Figure 5. Raman spectroscopy in InAs nanowire arrays. (a) Typical spectrum of the InAs nanowires on $\mathrm{Si}$, (b) evolution of the intensities of the peaks corresponding to InAs and $\mathrm{Si}$ and (c) normalized values of the volume of a single nanowire $\left(V_{\mathrm{NW}}\right)$, equivalent thin film thickness of the array $\left(V_{\text {InAs }}\right)$ as well as the surface covering of the nanowire array $\left(S_{\text {InAs }}\right)$ and single nanowire $\left(S_{\mathrm{NW}}\right)$.

the whole nanowire volume even though the strong absorption of InAs at $488 \mathrm{~nm}$ (absorption length is about $8 \mathrm{~nm}$ ). In Raman spectroscopy, not only enhancement for incident light 
is important, but also how the scattered light interacts with the nanowire and should be taken into account [69-71]. It has been shown in previous works that light extraction efficiency in an array can be explained by the Maxwell-Garnett effective medium approximation [72]. Under such framework, arrays with smaller nanowire density present an effective refractive index closer to that of air, thus enabling a better light extraction. However, the light resonances at the nanoscale should be also taken into account to have a full understanding of the Raman signal.

Similarly, the TO intensity also shows a change in the intensity decay as a function of the pitch for pitch distances around $800 \mathrm{~nm}$. However, contrary to the LO mode, the TO intensity decreases back again for large pitch distances (larger than $1200 \mathrm{~nm}$ ). In wurtzite material, it has been observed by Raman scattering that light coupling enables light entering the nanowires to a considerable extent through their side facets even for normal incidence and small bandgap material [73, 74]. Because the Raman signal is directly related to the electric field direction through the symmetry of the vibrational mode in question, the change in the field direction at the nanoscale can completely modify the Raman selection rules for different modes from the macroscopic point of view. Our results thus seem to indicate a similar effect, where the electromagnetic resonances in the nanowire favor symmetry conditions for extraction efficiency of the LO scattered light. This opens the way for fundamental studies in the area of LO phonon-electron/hole scattering in doped nanowires [75, 76].

\section{Conclusions}

In conclusion we have demonstrated growth of self-catalyzed InAs and GaAs nanowire arrays on a (111)Si substrate and explained some of the challenges associated with obtaining high yield. We have also explored the concept of ideal nanowire array configuration for optimal light absorption. In particular, we have shown how planar light waves incident perpendicularly to the substrate can exhibit resonances for certain nanowire diameters. This results in a self-concentration effect and a several-fold enhancement in the absorption rate. This phenomenon can be very useful in photovoltaic and light emitting devices. Finally, we show how the occurrence of absorption resonances in nanowires can lead to an enhancement of the LO phonon scattering, which can be of fundamental importance for the study of structural and functional properties of nanowires by Raman spectroscopy.

\section{Acknowledgments}

The authors thank funding through ERC Starting grant UpCon, FP7 Nanoembrace, Eranet-Russia InCoSiN, SNF funding and QSIT. SCB thanks funding through the SNF Marie Heim-Vögtlin program. EAL acknowledges Marie Curie EMMM3 fellowship.

\section{References}

[1] Yan P, Gargas D and Yang P D 2009 Nanowire photonics Nature Photon. 3 569-76
[2] Duan X, Huang Y, Cui Y, Wang J and Lieber C M 2001 Indium phosphide nanowires as building blocks for nanoscale electronic and optoelectronic devices Nature 40966

[3] Schmidt V, Riel H, Senz S, Karg S, Riess W and Gösele U 2006 Realization of a silicon nanowire vertical surround-gate field-effect transistor Small 2 85-8

[4] Kayes B M, Atwater H A and Lewis N S 2005 Comparison of the device physics principles of planar and radial p-n junction nanorod solar cells J. Appl. Phys. 97114302

[5] Chan C K, Peng H, Liu G, McIlwrath K, Zhang X F, Huggins R A and Cui Y 2008 High performance lithium battery anodes using silicon nanowires Nature Nanotechnol. 3 31-5

[6] Utama M I B, Zhang Q, Zhang J, Yuan Y, Belarre F, Arbiol J and Xiong O 2013 Recent developments and future directions in the growth of nanostructures by van der Waals epitaxy Nanoscale 5 3570-88

[7] Fontcuberta i Morral A, Spirkoska D, Arbiol J, Heigoldt M, Morante J R and Abstreiter G 2008 Prismatic quantum heterostructures synthesized on molecular-beam epitaxy GaAs nanowires Small 4 899-903

[8] Plissard S, Slapak D R, Verheijen M A, Hocevar M, Immink G W G, van Weperen I, Nadj-Perge S, Frolov S M, Kouwenhoven L P and Bakkers E P A M 2012 From InSb nanowires to nanocubes: looking for the sweet spot Nano Lett. 12 1794-8

[9] Dick K A, Deppert K, Mårtensson T, Seifert W and Samuelson L 2004 Growth of GaP nanotree structures by sequential seeding of 1D nanowires J. Cryst. Growth 272 131-7

[10] Cuscunà M, Convertino $\mathrm{A}$, Zampetti E, Macagnano A, Pecora A, Fortunato G, Felisari L, Nicotra G, Spinella C and Martelli F 2012 On-chip fabrication of ultrasensitive $\mathrm{NO}_{2}$ sensors based on silicon nanowires Appl. Phys. Lett. 101103101

[11] Conesa-Boj S et al 2012 Vertical 'III-V' V-shaped nanomembranes epitaxially grown on a patterned $\mathrm{Si}[001]$ substrate and their enhanced light scattering ACS Nano 6 10982-91

[12] Gudiksen M S et al 2002 Growth of nanowire superlattice structures for nanoscale photonics and electronics Nature 415 617-20

[13] Lauhon L J et al 2002 Epitaxial core-shell and core-multishell nanowire heterostructures Nature 420 57-61

[14] Chuang L C et al 2007 Critical diameter for III-V nanowires grown on lattice-mismatched substrates Appl. Phys. Lett. 90043115

[15] Hocevar M, Immink G, Verheijen M, Akopian N, Zwiller V, Kouwenhoven L and Bakkers E P A M 2012 Growth and optical properties of axial hybrid III-V/silicon nanowires Nature Commun. 31266

[16] Hillerich K, Dick K A, Wen C Y, Reuter M C, Kodambaka S and Ross F M 2013 Strategies to control morphology in hybrid group III-V/Group IV heterostructure nanowires Nano Lett. 13 903-8

[17] Conesa-Boj S, Dunand S, Russo-Averchi E, Heiss M, Rüffer D, Wyrsch N, Ballif C and Fontcuberta i Morral A 2013 Hybrid axial and radial $\mathrm{Si} / \mathrm{GaAs}$ heterostructures in nanowires Nanoscale 5 9633-9

[18] Bessire C D et al 2011 Trap-assisted tunneling in Si-InAs nanowire heterojunction tunnel diodes Nano Lett. 11 4195-9

[19] Tomioka K et al 2012 A III-V nanowire channel on silicon for high-performance vertical transistors Nature $\mathbf{4 8 8} 189$

[20] Lapierre R R et al 2013 III-V nanowire photovoltaics: review of design for high efficiency Phys. Status Solidi RRL 7 815-30 
[21] Kandala A, Betti T and Fontcuberta i Morral A 2009 General theoretical considerations on nanowire solar cell designs Phys. Status Solidi a 206 173-8

[22] Kelzenberg M D, Boettcher S W, Petykiewicz J A, Turner-Evans D B, Putnam M C, Warren M L, Spurgeon J M, Briggs R M, Lewis N S and Atwater H A 2010 Enhanced absorption and carrier collection in Si wire arrays for photovoltaic applications Nature Mater. 9 239-44

[23] Wallentin J et al 2013 InP nanowire array solar cells achieving $13.8 \%$ efficiency by exceeding the ray optics limit Science 339 1057-60

[24] Krogstrup P, Jørgensen H I, Heiss M, Demichel O, Holm J V, Aagesen M, Nygard J and Fontcuberta i Morral A 2013 Single-nanowire solar cells beyond the Shockley-Queisser limit Nature Photon. 7 306-10

[25] Borgström M T, Immink G, Ketelaars B, Algra R and Bakkers E P A M 2007 Synergetic nanowire growth Nature Nanotechnol. 2 541-4

[26] Dubrovskii V G et al 2012 Narrowing the length distribution of Ge nanowires Phys. Rev. Lett. 108105501

[27] Consonni V et al 2012 Quantitative description for the growth rate of self-induced GaN nanowires Phys. Rev. B 85155313

[28] Ramdani M R et al 2013 Arsenic pathways in self-catalyzed growth of GaAs nanowires Cryst. Growth Des. 12 91-6

[29] Bauer B et al 2010 Position controlled self-catalyzed growth of GaAs nanowires by molecular beam epitaxy Nanotechnology 21435601

[30] Fan H J, Werner P and Zacharias M 2006 Semiconductor nanowires: from self-organization to patterned growth Small 2 700-17

[31] Lerose D et al 2010 Ordered arrays of epitaxial silicon nanowires produced by nanosphere lithography and chemical vapor deposition original J. Cryst. Growth 312 2887-91

[32] Dick K A, Deppert K, Karlsson L S, Seifert W, Wallenberg L R and Samuelson L 2006 Position-controlled interconnected InAs nanowire networks Nano Lett. $62842-7$

[33] Pierret A, Hocevar M, Diedenhofen S L, Algra R E, Vlieg E, Timmering E C, Verschuuren M A, Immink G W G, Verheijen M A and Bakkers E P A M 2010 Generic nano-imprint process for fabrication of nanowire arrays Nanotechnology 21065305

[34] Subannajui K, Güder F and Zacharias M 2011 Bringing order to the world of nanowire devices by phase shift lithography Nano Lett. 11 3513-8

[35] Dick K A 2008 A review of nanowire growth promoted by alloys and non-alloying elements with emphasis on Au-assisted III-V nanowires Prog. Cryst. Growth Charact. Mater. 54 138-73

[36] De la Mata M et al 2012 Polarity assignment in ZnTe, GaAs, $\mathrm{ZnO}$, and $\mathrm{GaN}-\mathrm{AlN}$ nanowires from direct dumbbell analysis Nano Lett. 12 2579-86

[37] Tomioka K, Motohisa J, Hara S and Fukui T 2008 Control of InAs nanowire growth directions on Si Nano Lett. $83475-80$

[38] Uccelli E et al 2011 Three-dimensional multiple-order twinning of self-catalyzed GaAs nanowires on Si substrates Nano Lett. 11 3827-32

[39] Russo-Averchi E, Heiss M, Michelet L, Krogstrup P, Nygard J, Magen C, Morante J R, Uccelli E, Arbiol J and Fontcuberta i Morral A 2012 Suppression of three-dimensional twinning for a $100 \%$ yield of vertical GaAs nanowires on silicon Nanoscale 4 1486-90

[40] de la Mata M, Zhou X, Furtmayr F, Teubert J, Gradecak S, Eickhoff M, Fontcuberta i Morral A and Arbiol J 2013 A review of MBE grown 0D, 1D and 2D quantum structures in a nanowire J. Mater. Chem. $14300-12$
[41] de la Mata M et al 2013 Self-assembled semiconductor 0D, 1D and 2D quantum structures in a nanowire Mater. Today $16213-9$

[42] de la Mata M et al 2012 Polarity assignment in ZnTe, GaAs, $\mathrm{ZnO}$ and $\mathrm{GaN}-\mathrm{AlN}$ nanowires from direct dumbbell analysis Nano Lett. 12 2579-86

[43] Colombo C, Spirkoska D, Frimmer M, Abstreiter G and Fontcuberta i Morral A 2008 Ga-assisted catalyst-free growth mechanism of GaAs nanowires by molecular beam epitaxy Phys. Rev. B 77155326

[44] Dimakis E, Lähnemann J, Jahn U, Breuer S, Hilse M, Geelhaar L and Riechert H 2011 Self-assisted nucleation and vapor solid growth of InAs nanowires on bare $\mathrm{Si}(111)$ Cryst. Growth Des. 11 4001-8

[45] Mandl B, Dey A W, Stangl J, Cantoro M, Wernersson L E, Bauer G, Samuelson L, Deppert K and Thelander C 2011 Self-seeded, position-controlled InAs nanowire growth on Si: a growth parameter study J. Cryst. Growth $\mathbf{3 3 1} 51$

[46] Hertenberger S, Rudolph D, Bichler M, Finley J J, Abstreiter G and Koblmüller G 2010 Growth kinetics in position-controlled and catalyst-free InAs nanowire arrays on $\mathrm{Si}(111)$ grown by selective area molecular beam epitaxy J. Appl. Phys. 108114316

[47] Plissard S, Dick K A, Larrieu G, Godey S, Addad A, Wallart X and Caroff P 2010 Gold-free growth of GaAs nanowires on silicon: arrays and polytypism Nanotechnology 21385602

[48] Gibson S and Lapierre R 2013 Study of radial growth in patterned self-catalyzed GaAs nanowire arrays by gas source molecular beam epitaxy Phys. Status Solidi RRL 7 845-9

[49] Plissard S, Larrieu G, Wallart X and Caroff P 2011 High yield of self-catalyzed GaAs nanowire arrays grown on silicon via gallium droplet positioning Nanotechnology 22275602

[50] Russo-Averchi E, Dalmau-Mallorquí A, Canales-Mundet I, Tütüncüoğlu G, Alarcon-Lladó E, Heiss M, Rüffer D, Conesa-Boj S, Caroff P and Fontcuberta i Morral A 2013 Growth mechanisms and process window for InAs $\mathrm{V}$-shaped nanoscale membranes on $\mathrm{Si}[001]$ Nanotechnology 24435603

[51] Bauer B, Rudolph A, Soda M, Fontcuberta i Morral A, Zweck J, Schuh D and Reiger E 2010 Position controlled self-catalyzed growth of GaAs nanowires by molecular beam epitaxy Nanotechnology 21435601

[52] Colombo C et al $2008 \mathrm{Ga}$-assisted catalyst-free growth mechanism of GaAs nanowires by molecular beam epitaxy Phys. Rev. B 77155326

[53] Matteini F et al 2013 submitted

[54] Oskooi A F et al 2010 A flexible free-software package for electromagnetic simulations by the FDTD method Comput. Phys. Commun. 181 687-702

[55] Kupec $\mathrm{J}$ et al 2010 Light absorption and emission in nanowire array solar cells Opt. Express 18 27589-605

[56] Heiss M and Fontcuberta i Morral A 2012 Fundamental limits in the external quantum efficiency of single nanowire solar cells Appl. Phys. Lett. 99263102

[57] Cao L Y et al 2011 Optical coupling of deep-subwavelength semiconductor nanowires Nano Lett. 11 1463-8

[58] Colombo C et al 2011 Engineering light absorption in single-nanowire solar cells with metal nanoparticles New J. Phys. 13123026

[59] Cao L Y et al 2009 Engineering light absorption in semiconductor nanowire devices Nature Mater. 8 643-9

[60] Jain P K, Lee K S, El-Sayed I H and El-Sayed M A 2006 Calculated absorption and scattering properties of gold nanoparticles of different size, shape, and composition: applications in biological imaging and biomedicine J. Phys. Chem. B $1107238-48$

[61] Muskens O L, Del Fatti N, Vallee F, Huntzinger J R, Billaud P and Broyer M 2006 Single metal nanoparticle absorption 
spectroscopy and optical characterization Appl. Phys. Lett. 88063109

[62] Shockley W and Queisser H J 1961 Detailed balance limit in efficiency of pn junction solar cells J. Appl. Phys. 32510

[63] Kelzenberg M D et al 2010 Enhanced absorption and carrier collection in Si wire arrays for photovoltaic applications Nature Mater. 9 239-44

[64] Trevino J et al 2012 Plasmonic-photonic arrays with aperiodic spiral order for ultra-thin film solar cells Opt. Express 20 A418-30

[65] Hoermann N G et al 2011 Effects of stacking variations on the lattice dynamics of InAs nanowires Phys. Rev. B 84155301

[66] Kusch P et al 2012 Band gap of wurtzite GaAs: a resonant Raman study Phys. Rev. B 86075317

[67] Wang P et al 2012 Conduction band structure in wurtzite GaAs nanowires: a resonant Raman scattering study Appl. Phys. Lett. 100073102

[68] Panda J K et al 2012 Raman sensitivity to crystal structure in InAs nanowires Appl. Phys. Lett. 100143101

[69] Cao L, Laim L, Valenzuela P D, Nabet B and Spanier J E 2007 On the Raman scattering from semiconducting nanowires J. Raman Spectrosc. 38697

[70] Panda J K, Roy A, Singha A, Gemmi M, Ercolani D, Pellegrini V and Sorba L 2013 Internal field induced enhancement and effect of resonance in Raman scattering of InAs nanowires Solid State Commun. 16026
[71] Lopez F J, Hyon J K, Givan U, Kim I S, Holsteen A L and Lauhon L J 2012 Diameter and polarization-dependent Raman scattering intensities of semiconductor nanowires Nano Lett. 122266

[72] Henneghien A L, Tourbot G, Daudin B, Lartigue O, Desieres Y and Gerard J M 2011 Optical anisotropy and light extraction efficiency of MBE grown GaN nanowires epilayers Opt. Express 19527

[73] Lazić S, Gallardo E, Calleja J M, Agulló-Rueda F, Grandal J, Sánchez-Garcia M A, Calleja E, Luna E and Trampert A 2007 Phonon-plasmon coupling in electron surface accumulation layers in InN nanocolumns Phys. Rev. B 76205319

[74] Pfuller C, Ramsteiner M, Brandt O, Grosse O, Rathsfeld A, Schmidt A, Geelhaar L and Riechert H 2012 Raman spectroscopy as a probe for the coupling of light into ensembles of sub-wavelength-sized nanowires Appl. Phys. Lett. 101083104

[75] Ketterer B et al 2012 Mobility and carrier density in p-type GaAs nanowires measured by transmission Raman spectroscopy Nanoscale 41789

[76] Ketterer B et al 2011 Phonon confinement and plasmon-phonon interaction in nanowire-based quantum wells Phys. Rev. B 83245327 